\title{
Copy editing
}

Public Domain

\section{Source}

\section{Open Research Glossary.}

A type of editing designed to improve the formatting, style, and accuracy of text. It usually does not involve changing the content of the original text. 